occur at all seasons, it is more so in the hot weather than in the cold ; but the rainy season is that in which this complication supervenes most frequently, runs its course towards destruction of the eye most rapidly, and is least amenable to treatment. But though the hot weather undoubtedly exercises an unfavourable, and the rains a pernicious, influence in inducing this complication, the primary and most important cause of its occurrence is to be found in the wretchedly nourished state in which the majority of the patients come into the hospital. Were it practicable, it would be extremely advisable that most of the patients who present themselves suffering from cataract should be subjected to preparatory treatment, with a view to improving their health previous to operating. To anyone acquainted with the native character and the working of Indian civil hospitals the impracticability of carrying such a practice into effect will be apparent. A most characteristic feature in the natives is the scepticism as to one's surgical skill that is engendered in his mind by any delay in operating. It is common in Indian hospitals to meet with patients who, on admission, are solicitous to be put on the operating-table, but when asked to remain in hospital to undergo treatment preparatory to the operation, lose all confidence in the surgeon, and abscond under the impression that the delay in operating is due to doubt on the part of the surgeon as to the prospect of a successful result ensuing.

When suppuration has once set in it is rare to find a case recover, sloughing of the cornea or suppuration of the deep parts of the eyeball following as a general rule. In some few cases, however, in which the suppuration was confined to the seat of the corneal incision, the patients recovered with useful vision. My experience is that when there is any tendency to suppuration any mode of treatment depressing to the system is to be deprecated. On the contrary, under such circumstances, a tonic and stimulating treatment should be had recourse to at an early date. There is no doubt that the most successful way of combating this dire complication is to improve the health of the patient before undertaking the operation, but when this is impracticable the patient should be placed on a liberal diet with alcoholic stimulants, and have a tonic of quinine and iron administered to him from the day he enters hospital. In addition to these measures, when any tendency to suppuration displays itself I am in the habit of ordering hot poppy fomentations for half an hour three times daily, and a large pad with firm bandaging to the eye during the intervals, and $I$ think that in this way I have saved many an eye.

Next to suppuration of the cornea the complication which occurred most frequently after the operation was iritis. Indeed, with the exception of about a dozen cases in which no vision resulted, owing to internal hæmorrhage, large escape of vitreous, or some previously existing deep-seated disease of the eye, all my unsuccessful results were attributed to these two sequelæe. As might be expected from the description of the patients which I have already given, the variety of iritis that occurs is generally of a low type, and if not quickly checked has a tendency to terminate in suppuration. In order to guard against the supervention of this complication great care in the performance of the iridectomy is essential. The most important points to be attended to are that the iris should be withdrawn outside the corneal wound very gently, and not put on the stretch ; that the excision should extend through the whole width of the iris, leaving behind a mere slit only; and that the extremities of the divided iris be carefully replaced within the aqueous chamber, so that they may not be in. carcerated between the lips of the corneal wound. When, in spite of all precautions, iritis does supervene, the measures I have found most effective in the treatment of this complication are, the introduction into the eye of a drop or two of sulphate of atropine solution (four grains to the ounce) three or four times in the twenty-four hours, in addition to the external application of extract of belladonna round the orbit; the administration of morphia both internally and by hypodermic injection, to relieve the pain ; complete avoidance of exposure of the eye to light; and the administration of iodide of potassium in five-grain doses three times a day. Formerly, in cases of iritis supervening, in strong persons, after the operation of extraction of the lens, I invariably administered mercury, but I have not found its exhibition in such cases attended by the happy results that generally follow its administration in cases of ordinary idiopathic or traumatic iritis. I now almost invariably give the iodide of potash in cases of iritis following the operation of cataract extraction, and consider that it is far superior to mercury in preventing effusion of lymph and in facilitating its absorption.

\section{DEATH FROM EXTRA-UTERINE PREGNANCY SIMULATING ACUTE IRRITANT POISONING.}

\section{BY FREDERICK A. GRAY, L.R.C.P. LOND., M.R.C.S.}

THE following case seems to be worth recording on account of its having given rise to a suspicion of poisoning. The chief points in the case appear to be-Firstly, the suddenness of the onset of symptoms soon after a meal. secondly, their rapidly fatal character; thirdly, the severe vomiting; and, fourthly, the severe purging, which has rarely, if ever, been described as occurring in similar cases. These symptoms, occurring in an apparently healthy young woman, were so suspicious that the coroner considered an inquest necessary, and makes the case one of some toxicological interest.

S. IL—, aged twenty-six years, was a married woman, and has had four children; was last confined one year and nine months ago ; has been suckling the child ever since. Her husband believes she has seen her courses for several months past irregularly. On January 4th, 5th, and 6th there was a bloody discharge from the uterus, and she thought her courses had come on. On the afternoon of the 6th she said she felt something in her stomach, but she seemed to be in her usual health and said nothing more about it. On the 7th she took some broth at about 8 P.M.; at 9 P.M. she said she felt a pain in her stomach. This was quickly followed by vomiting and purging, which increased in severity. She died at about 11 P.M. in a state of collapse. I was sent for, but found life extinct on arriving. As I could not give a certificate of the cause of death, an inquest was decided on by the coroner, after some delay.

A post-mortem examination was made on the 12 th, the weather in the interval being dry and frosty. The body was found well nourished. Rigor mortis was well-marked. There were no marks of any kind about the body, mucous membrane of mouth, \&c. On opening the abdomen a large clot of blood became visible at the lower part, covering the great omentum. There was no evidence of inflammation. The bowels were collected in coils close to the spine, and were perfectly empty, except that they contained some round worms. The lower part of the abdominal and the pelvic cavities were filled with blood, measuring about two quarts. On examining the pelvis carefully, the uterus was found to be slightly enlarged; the ovaries were normal in size and position. On the right side, however, apparently in the broad ligament, was found a mass about the size of a walnut. The pelvic contents were removed altogether, and examined. The tumour mentioned above was found to be connected with the Fallopian trbe, about an inch and a quarter from its fimbriated extremity. A bristle passed readily down the tube, till it was arrested by the mass. The mass consisted of blood-clot, over which the Fallopian tube was so stretched in parts that its structure could hardly be recognised. Unluckily the parts were preserved in spirit for some days, so that when the tumour was examined the foetus could not be made out among the clots of blood, though in parts villi were plainly recognisable. The veins in the immediate neighbourhood of the tumour and in the broad ligament were much enlarged. At about the middle of the mass, at the back, was a hole of the size of a pea, with ragged edges, through which the blood found in the abdomen had evidently been poured forth. On opening the enlarged aterus the lining membrane was found thickened, and there was a small quantity of blood-stained mucus. The openings of the two Fallopian tubes were visible, and a bristle passed readily through the left, but was arrested on the right by the mass. The ovaries were normal, and the right had a well-marked corpus luteum. The large vessels in the abdomen were quite natural. All the other organs were healthy. The brain was not examined.

Ottery St. Mary. 